\title{
Relationship Between Gross Motor Function and Daily Functional Skill in Children With Cerebral Palsy
}

\author{
Tae Gun Kwon, $\mathrm{MD}^{1}$, Sook-Hee Yi, $\mathrm{MD}^{1}$, Tae Won Kim, $\mathrm{MD}^{1}$, \\ Hyun Jung Chang, $\mathrm{MD}^{2}$, Jeong-Yi Kwon, $\mathrm{MD}^{1}$
}

\begin{abstract}
${ }^{1}$ Department of Physical and Rehabilitation Medicine, Samsung Medical Center, Sungkyunkwan University School of Medicine, Seoul; ${ }^{2}$ Department of Physical Medicine and Rehabilitation, Samsung Changwon Hospital, Sungkyunkwan University School of Medicine, Changwon, Korea
\end{abstract}

\begin{abstract}
Objective To investigate the relationship between gross motor function and daily functional skill in children with cerebral palsy (CP) and to explore how this relationship is moderated by the Gross Motor Function Classification System, Bimanual Fine Motor Function (BFMF), neuromotor types, and limb distribution of CP.

Methods A cross-sectional survey of 112 children with CP (range, 4 years to 7 years and 7 months) was performed. Gross motor function was assessed with the Gross Motor Function Measure-66 (GMFM-66) and functional skill was assessed with the Pediatric Evaluation of Disability Inventory-Functional Skills Scale (PEDI-FSS).

Results GMFM-66 scores explained 49.7\%, 67.4\%, and $26.1 \%$ of variance in the PEDI-FSS scores in the self-care, mobility, and social function domains, respectively. Significant moderation by the distribution of palsy and BFMF classification levels II, III, and IV was found in the relationship between GMFM-66 and PEDI-FSS self-care. Further significant moderation by the distribution of palsy was also observable in the relationship between GMFM-66 and PEDI-FSS mobility.

Conclusion These findings suggest that limb distribution and hand function must be considered when evaluating gross motor function and functional skills in children with $\mathrm{CP}$, especially in unilateral CP.
\end{abstract}

Keywords Disability evaluation, Severity of illness index, Cerebral palsy, Child

Received February 20, 2012; Accepted September 3, 2012

Corresponding author: Jeong-Yi Kwon

Department of Physical and Rehabilitation Medicine, Samsung Medical Center, Sungkyunkwan University School of Medicine, 81 Irwon-ro, Gangnam-gu, Seoul 135-710, Korea

Tel: +82-2-3410-2818, Fax: +82-2-3410-0052, E-mail: jeongyi@gmail.com

(a) This is an open-access article distributed under the terms of the Creative Commons Attribution Non-Commercial License (http://creativecommons. org/licenses/by-nc/3.0) which permits unrestricted noncommercial use, distribution, and reproduction in any medium, provided the original work is properly cited.

Copyright $\odot 2013$ by Korean Academy of Rehabilitation Medicine

\section{INTRODUCTION}

Cerebral palsy (CP) is defined as 'a group of permanent disorders of the development of movement and posture, causing activity limitation, that are attributed to nonprogressive disturbances that occurred in the developing fetal or infant brain.' The motor disorders present in CP are often accompanied by disturbances of sensation, perception, cognition, communication, and behavior, by epilepsy and secondary musculoskeletal problems [1]. This definition includes the concept of activity limitation, 
which is the term used in the International Classification of Functioning, Disability, and Health (ICF), as defined by the World Health Organization (WHO) in $2001[2,3]$.

However, since motor disability related with sitting, standing, walking and running appears as the main symptom in children with $\mathrm{CP}$, the assessment and treatment of CP have focused on gross motor function (capacity). The Gross Motor Function Measure (GMFM) designed by Russell et al. [4] in 1993 is used to measure the level of motor development and changes of gross motor function in a standardized environment in children with CP. GMFM-66 is comprised of a subset of the 88 items identified (through Rasch analysis) as contributing to the measure of gross motor function in children with $\mathrm{CP}$, ranging from 0 (lowest motor function) to 100 (highest motor function).

The Pediatric Evaluation of Disability Inventory (PEDI) designed by Haley et al. [5] is used to measure if a child can perform certain activities (capability) and carries out these activities (performance) in daily environments such as home, kindergarten or school, rather than measuring capacity in standardized environments. Capability and performance in real-life situations, as defined by the WHO, are reflected in the PEDI's evaluation items, including communication, self-care management and getting in and out of cars. In recent years, the PEDI has been widely used to evaluate the daily functional skills in children with CP for clinical and experimental purposes $[6,7]$.

Several studies have explored the relationship between the GMFM and the PEDI in order to identify whether improvement in gross motor function is actually related with improvement in daily functional skills. Smits et al. [8] reported that the GMFM- 66 explained $90 \%$ of variables in the mobility domain of the PEDI in 116 children with $\mathrm{CP}$ aged between 4 years and 8 months and 7 years and 7 months. Holsbeeke et al. [9] reported that the correlations between motor capacity, motor capability, and motor performance were high, between 0.84 and 0.92 , and significant $(\mathrm{p}<0.001)$ in children with $\mathrm{CP}$ aged 30 months on average. However, when comparing children with the same level of motor capacity or capability, large ranges at the level of motor performance were found. Tieman et al. [10] also reported statistically significant difference in performance across multiple settings for children with $\mathrm{CP}$ in all capability groups. As gross motor function (capacity) and functional skill (capability or performance) do not always correlate with each other, examination of a child's capability and performance in the settings that are important to the child's daily life, such as home, school and outdoors, needs to be considered.

As most previous studies have compared the gross motor function only with the mobility domain of the PEDI, studies addressing the relationship between the gross function and other domains of functional skills (self-care, social function) that can be affected by the gross motor function have been limited. To our knowledge, only one study by Ostensjo et al. [11] has compared gross motor function with the domains of self-care, mobility, and social function in 95 children with CP aged between 25 and 87 months. In spite of children with CP comprising a very heterogeneous group, this study was not conducted based on the Gross Motor Function Classification System (GMFCS). As such, understanding the correlation between the GMFM and the PEDI by the severity of CP was limited.

The development of daily functional skills is affected by personal, social, and cultural factors. Jeong et al. [12] conducted a study into the influence of culture on PEDI scores. This study reported that the PEDI scores in Korean children were lower than those in American children in some age groups. This result implied that there may exist some differences in correlation between the capacity and capability of Korean children with CP.

Independent performance is also affected by fine motor function as well as gross motor function in everyday life. Hand function, expressed by fine motor function, is essential in handling objects in daily life. The Bimanual Fine Motor Function (BFMF, Appendix 1) classification system, which corresponds to the GMFCS levels, has been developed for the evaluation of hand function by severity in children with CP [13]. Various studies have revealed that a lack of development in hand function causes a decline in sensory information input, voluntary play, experience, and learning ability. That is, it leads to a functional decline in daily life and social participation restrictions [14]. Decline in fine motor function is frequently observed in children with CP. As such, fine motor function needs to be considered along with gross motor function to more fully comprehend all domains of the children's daily lives.

From this perspective, the aim of this study is to investigate the relationship between gross motor function and all domains of daily functional skills in the South Korean 
children with CP, by comparing GMFM-66 and PEDI scores. It also aims to explore the factors which affect the relationship between these measures by analyzing the moderation effects of the neuromotor type, distribution, severity, and hand function in children with CP.

\section{MATERIALS AND METHODS}

\section{Method}

The present study was conducted in the form of a retrospective chart review, as approved by the Institutional Review Board of the Samsung Medical Center. The diagnoses, neuromotor types, distribution of palsy and GMFCS levels of children with CP were recorded by an experienced pediatric physiatrist. GMFM was evaluated by three trained physical therapists who specialize in children, using translated GMFM evaluation sheets in a physical therapy room free of disturbance [4]. PEDI evaluations were conducted by two trained pediatric occupational therapists through structured interviews with the children's parents or persons who were familiar with the children, using the Korean version of the PEDI evaluation sheet translated by Jeong et al. [12] BFMF evaluations for assessing hand function were also conducted by two trained pediatric occupational therapists.

\section{Subjects}

Children with CP aged 4 to 7 years who had visited the outpatient clinic of the Department of Physical and Rehabilitation Medicine at the Samsung Medical Center, Seoul, Republic of Korea, between February 2009 and September 2011, were screened. Through the hospital database system, 145 children aged 4 to 7 years who had performed GMFM as well as PEDI evaluation within the past two weeks were initially selected. After reviewing subjects' medical records, 10 subjects who had genetic disease, 18 who had undergone botulinum toxin injection within the previous three months, and 5 who had undergone orthopedic or neurosurgical operations within the past year were excluded. Thus a total of 112 children with CP were selected for final analysis. The BFMF classification could be obtained for 55 out of the 112 final subjects. The age of the subjects ranged from 4 years to 7 years and 9 months, and the mean age was $5.8 \pm 1.5$ years. As for the neuromotor types and the distribution of palsy, the spastic type was the most frequent at 102 (91.1\%) subjects and of those, $83(81.4 \%)$ were bilateral (Table 1). By
GMFCS classification, level I was the most frequent with $32(28.6 \%)$ subjects and by BFMF classification, level II was the most frequent with 28 (50.9\%) subjects (Table 1 ).

\section{Data analysis}

Statistical analysis were conducted using SPSS ver. 19.0 (SPSS Inc., Chicago, IL, USA), and a p-value of $<0.05$ was considered statistically significant. The data was analyzed as follows, in light of both the purposes of this study and the characteristics of the variables.

Univariate regression analysis was used to investigate the relationship between the gross motor function (capacity) and the daily functional skill (capability) as the percentage of explained variance for each PEDIFunctional Skills Scales (PEDI-FSS) by the GMFM-66. We only used the PEDI-FSS to exclude the caregiver's assistance. The scaled PEDI scores used in this study reflect the children's functional skill, and these have been

Table 1. Characteristics of participants (n=112)

\begin{tabular}{|cc|}
\hline \multicolumn{1}{c}{ Characteristic } & Participant \\
\hline Age (yr) & $5.9 \pm 1.5$ \\
Sex & $64(57.1)$ \\
Male & $48(42.9)$ \\
\hline Female & \\
\hline Classification & \\
\hline Neuromotor type & $4(3.6)$ \\
\hline Dyskinetic & $6(5.4)$ \\
\hline Ataxic & $102(91.1)$ \\
\hline Spastic & \\
\hline GMFCS level & $32(28.6)$ \\
\hline I & $31(27.7)$ \\
\hline II & $28(25.0)$ \\
\hline III & $16(14.3)$ \\
\hline IV & $5(4.5)$ \\
\hline V & \\
\hline BFMF level & $6(10.9)$ \\
\hline I & $28(50.9)$ \\
\hline II & $9(16.4)$ \\
\hline III & $10(18.2)$ \\
\hline IV & $2(3.6)$ \\
\hline V & \\
\hline
\end{tabular}

$\overline{\text { Values are presented as number (\%) or mean } \pm \text { standard }}$ deviation.

GMFCS, Gross Motor Function Classification System; BFMF, Bimanual Fine Motor Function. 
transformed to a scale of 0 to 100 , where 0 represents no ability and 100 represents full capability in performing the functional skill items in a particular domain. One way analysis of variance (ANOVA) and post hoc analysis using the Scheffe method were calculated to evaluate the difference between GMFM-66 and PEDI-FSS, by GMFCS, BFMF, neuromotor type, and the distribution of palsy. Finally, multivariate regression analysis for the evaluation of moderation effects was carried out using GMFCS, BFMF, the neuromotor type, and the distribution of palsy as variables, to identify the factors which can affect the relationship between the GMFM-66 and the PEDI-FSS.

\section{RESULTS}

Relationship between the gross motor function and the daily functional skill

The results of the regression analysis showed that GMFM-66 scores explained $49.7 \%, 67.4 \%$, and $26.1 \%$ of the variance of PEDI-FSS self-care, mobility, and social function scores, respectively (Table 2).

Table 2. Association between PEDI-FSS and GMFM-66 by univariate linear regression analysis

\begin{tabular}{lccc}
\multicolumn{1}{c}{ Variable } & $\mathbf{R}^{2}$ & $\beta \pm \mathbf{S E}$ & $\mathbf{p}$-value \\
\hline PEDI-FSS & & & \\
Self-care & 0.497 & $0.72 \pm 0.06$ & $\mathrm{p}<0.001$ \\
Mobility & 0.674 & $0.98 \pm 0.05$ & $\mathrm{p}<0.001$ \\
Social function & 0.261 & $0.66 \pm 0.10$ & $\mathrm{p}<0.001$ \\
\hline
\end{tabular}

PEDI-FSS, Pediatric Evaluation of Disability InventoryFunctional Skills Scale; GMFM-66, Gross Motor Function Measure-66; $\beta$, slope; SE, standard error.
Differences in the gross motor function and daily functional skill by the characteristics of CP

Significant differences were observed in the mean of GMFM-66 scores and the PEDI-FSS scores by the neuromotor type and the distribution of palsy $(\mathrm{p}<0.05)$. Based on the post hoc analysis, GMFM-66 scores, and PEDI-FSS mobility scores were higher in children with unilateral spastic type compared to those with bilateral spastic, ataxic, and dyskinetic type (Table 3). Significant differences in the mean of the GMFM-66 and all domains of the PEDI-FSS were observed by GMFCS and BFMF classifications $(\mathrm{p}<0.05)$ (Table 1). Based on the post hoc analysis, significant differences in GMFM-66 scores were observed between each GMFCS level. Significant differences in PEDI-FSS self-care scores were observed between the individual GMFCS levels, except for between levels II and III. In the PEDI-FSS mobility domain, significant differences were observed between all GMFCS levels, except for between levels III and IV and between IV and V. Meanwhile, in the social function domain, significant differences were observed only between GMFCS level $\mathrm{V}$ and other levels (Table 1). Based on post hoc analysis by BFMF levels, significant differences in GMFM-66 scores were observed only between BFMF levels I, II, III and IV, V. For the PEDI-FSS self-care, significant differences were observed between each level except for between BFMF levels I and II. For the PEDI-FSS mobility, there were significant differences observed only between BFMF levels I, II, III and levels IV and V. Further, for the PEDI-FSS social function, significant differences were only observed between BFMF level I (or II) and level IV (or V), and level III and V (Table 1).

Table 3. GMFM-66 and PEDI-FSS stratified by neuromotor type and limb distribution

\begin{tabular}{lrlccc}
\hline & No. & GMFM-66 & \multicolumn{3}{c}{ PEDI-FSS } \\
\cline { 3 - 6 } & & & Self-care & Mobility & Social function \\
\hline Spastic & 102 & $62.94 \pm 17.84$ & $63.28 \pm 12.60$ & $59.39 \pm 20.32$ & $67.64 \pm 15.85$ \\
Bilateral & 83 & $58.77 \pm 15.71^{\text {a) }}$ & $62.43 \pm 12.85$ & $55.45 \pm 18.66^{\text {a) }}$ & $67.26 \pm 15.99$ \\
Unilateral & 19 & $81.18 \pm 15.15^{\text {b,c,d }}$ & $66.96 \pm 11.04$ & $76.62 \pm 18.58^{\mathrm{b}, \mathrm{c}, \mathrm{d})}$ & $69.29 \pm 15.52$ \\
Dyskinetic & 4 & $56.23 \pm 5.62^{\mathrm{a})}$ & $61.08 \pm 21.13$ & $62.05 \pm 16.32^{\mathrm{a})}$ & $72.38 \pm 27.61$ \\
Ataxic & 6 & $60.83 \pm 22.70^{\mathrm{a})}$ & $55.63 \pm 17.09$ & $37.87 \pm 26.80^{\mathrm{a})}$ & $54.82 \pm 16.64$ \\
Total & 112 & $62.59 \pm 17.76$ & $62.79 \pm 13.16$ & $58.33 \pm 20.96$ & $67.12 \pm 16.46$ \\
\hline
\end{tabular}

Values are presented as mean \pm standard deviation. $\mathrm{p}<0.05$.

GMFM-66, Gross Motor Function Measure-66; PEDI-FSS, Pediatric Evaluation of Disability Inventory-Functional Skills Scale; $\mathrm{CP}$, cerebral palsy.

Significantly different compared with ${ }^{\text {a) }}$ unilateral spastic $\mathrm{CP}$, ${ }^{\text {b) }}$ bilateral spastic $\mathrm{CP},{ }^{\mathrm{c})}$ dyskinetic $\mathrm{CP}$, and ${ }^{\mathrm{d})}$ ataxic $\mathrm{CP}$. 
The moderation effects of characteristics of CP on the relationship between gross motor function and daily functional skill

Significant moderation by the distribution of palsy and BFMF classification levels II, III and IV was found in the relationship between GMFM-66 and PEDI-FSS selfcare. Further significant moderation by the distribution of palsy was also observed for the relationship between GMFM-66 and PEDI-FSS mobility (Table 4). Additional univariate regression analysis within groups by distribution of palsy revealed that GMFM-66 scores explained only $29.7 \%$ of variance of PEDI-FSS self-care scores, $35.2 \%$ of variance of PEDI-FSS mobility scores in children with unilateral spastic $\mathrm{CP}$, and $61.7 \%$ and $78.2 \%$ in bilateral spastic CP, respectively.

\section{DISCUSSION}

In the area of pediatric rehabilitation, reliable and valid measurement tools can provide solid information for the establishment of therapeutic planning and outcome measure. Therefore, measurement tools should objectively reflect children's current levels of function. The GMFM measures what a child can do in standardized circumstances (capacity) and is formally administered by a pediatric therapist trained in its use. The PEDI evaluates what a child can do (capability) and does do (performance) in a more real-world environments, without or with assistance, and is completed through interviews with the caregivers. The PEDI indicates the child's adaptation or adjustment in motor functions in actual daily life, but takes a relatively long time to evaluate. As the GMFM and the PEDI provide different perspectives on a child's motor function and functional skill, it is important to comprehend the relationship between the results of both measurement tools.

This study showed that daily functional skill is related to gross motor function in Korean children with CP aged 4 to 7 years, explaining $49.7 \%, 67.4 \%$, and $26.1 \%$ of variance in the self-care, mobility, and social function

Table 4. Interaction terms of GMFM with GMFCS level, neuromotor type, limb distribution, and BFMF by multivariate analysis for moderation

\begin{tabular}{|c|c|c|c|c|}
\hline & \multicolumn{4}{|c|}{ PEDI-FSS } \\
\hline & \multicolumn{2}{|c|}{ Self-care } & \multicolumn{2}{|c|}{ Mobility } \\
\hline & $\beta \pm \mathbf{S E}$ & p-value & $\beta \pm \mathbf{S E}$ & p-value \\
\hline GMFM-66 & $0.65 \pm 0.06$ & $<0.001$ & $1.00 \pm 0.05$ & $<0.001$ \\
\hline GMFM-66 * GMFCS I (ref.cat) & - & - & - & - \\
\hline GMFM-66 * GMFCS II & $1.29 \pm 3.13$ & 0.680 & $-1.04 \pm 5.28$ & 0.844 \\
\hline GMFM-66 * GMFCS III & $5.56 \pm 2.18$ & 0.053 & $-0.36 \pm 3.08$ & 0.907 \\
\hline GMFM-66 * GMFCS IV & $2.51 \pm 6.02$ & 0.735 & $-12.10 \pm 10.50$ & 0.256 \\
\hline GMFM-66 * GMFCS V & $1.29 \pm 3.13$ & 0.680 & $-1.04 \pm 5.28$ & 0.844 \\
\hline GMFM-66 * Spastic (ref.cat) & - & - & - & - \\
\hline GMFM-66 * dyskinetic & $10.42 \pm 6.23$ & 0.110 & $11.65 \pm 7.27$ & 0.125 \\
\hline GMFM-66 * ataxic & $1.27 \pm 3.72$ & 0.737 & $-23.2 \pm 8.68$ & 0.114 \\
\hline GMFM-66 * unilateral (ref.cat) & - & - & - & - \\
\hline GMFM-66 * bilateral & $8.80 \pm 2.48$ & $0.001^{*}$ & $2.13 \pm 2.70$ & $0.043^{*}$ \\
\hline GMFM- 66 * BFMF I (ref.cat) & - & - & - & - \\
\hline GMFM-66 * BFMF II & $-3.26 \pm 3.23$ & $0.032^{*}$ & $-3.02 \pm 3.54$ & 0.400 \\
\hline GMFM- 66 * BFMF III & $-15.71 \pm 4.86$ & $0.008^{*}$ & $3.255 \pm 6.11$ & 0.605 \\
\hline GMFM- 66 * BFMF IV & $-16.96 \pm 6.59$ & $0.017^{*}$ & $-4.03 \pm 6.05$ & 0.515 \\
\hline GMFM- 66 * BFMF V & $-32.93 \pm 18.97$ & 0.143 & $28.63 \pm 18.10$ & 0.174 \\
\hline
\end{tabular}

GMFM-66, Gross Motor Function Measure-66; GMFCS, Gross Motor Functional Classification System; PEDI-FSS, Pediatric Evaluation of Disability Inventory-Functional Skills Scale; BFMF, Bimanual Fine Motor Function; $\beta$, slope; SE, standard error; ref.cat, reference category.

${ }^{*} \mathrm{p}<0.05$. 
domains of PEDI, respectively (Table 2). This is the first study performed in the Republic of Korea to have addressed the relationship between gross motor function and all domains of daily functional skill. In this study, a relatively weaker relationship between gross motor function and daily mobility skill was observed (67.4\%) than was found in studies conducted in the United States (90.0\%) [8] and Sweden (75.0\%) [11]. This difference has also been identified from a study in China, which showed a correlation coefficient of $(r=0.83-0.90)$ [15] between the GMFM-66 and PEDI-FSS mobility scores. These different results may be due to a translation gap that leads to obscurity of measurement criteria when the original items are applied, as well as differences in childcare cultures and social environments. However, items in the domain of mobility of the PEDI, such as toilet use, moving in a wheelchair and moving method and speed, have been found to show smaller differences in translation compared to items in the domain of self-care and social function. Jeong et al. [12] found that there were no significant differences between Korea and the United States in PEDI scores for the mobility domain in children aged 4 years or older. Therefore, the relatively weaker relationship between mobility and gross motor function found in this study is considered to have been affected by cultural and environmental differences rather than by a translation gap between the PEDI evaluation sheets.

The self-care and mobility domains of the PEDI-FSS showed significant differences by GMFCS levels, in contrast to the domain of social function. The low correlation for the latter function can be explained in that rates of low cognitive function (intellectual disability) vary significantly between levels of CP and that levels of impairment vary within cerebral palsy subtypes [16]. In addition social function is affected by various multifactor causes such as education and economic and social factors as well as by motor function. Meanwhile, by BFMF classification, significant differences in PEDI-FSS mobility were observed only between BFMF levels I, II, III and levels IV and V. This result shows that the ability of the child to hold and release objects with only one hand and to be able to manipulate objects (as based on the BFMF classification) serves as an important factor in the mobility of daily living.

In the PEDI-FSS self-care domain, significant differences were observed between each BFMF level, except for levels I and II in this study. Ahn et al. [17] previously observed statistically significant differences at all levels for the correlations between the Manual Ability Classification System (MACS) and PEDI-FSS self-care. These conflicting results suggest that two-hand function classification systems are not always consistent. The MACS classifies children based on how they use both hands when they deal with objects in daily life and as such might better reflect actual functional skills. Further research may be warranted to investigate the comparison between the two classification systems.

Significant moderation by the distribution of palsy and the BFMF classification levels II, III, and IV was found in the relationship between GMFM-66 and PEDI-FSS selfcare. Further significant moderation by the distribution of palsy was also observed in the relationship between GMFM-66 and PEDI-FSS mobility. In the mobility domain of PEDI, when the subjects were limited to spastic children with CP, GMFM-66 scores explained $78.2 \%$ in children with bilateral spastic $\mathrm{CP}$, which was much higher than the $35.2 \%$ for children with unilateral spastic CP. This result is consistent with previous findings from studies performed in the United States [8]. These findings may have implications for goal-setting to enhance the daily functional skill of children with $\mathrm{CP}$. As the gross motor function is transferred to real-life daily functional skill in bilateral spastic children with $\mathrm{CP}$, treatment and assessment needs to focus on gross motor function enhancement. Conversely in unilateral spastic children with $\mathrm{CP}$, treatment and assessment should focus on continuous situation training [8].

In children with $\mathrm{CP}$, the domain of self-care in daily life is closely related with their hand fine motor function [13,17-19]. In a study by Ahn et al. [17] that examined children with spastic $\mathrm{CP}$, the self-care domain of the PEDI showed high correlations with the MACS. Our study found that BFMF classification significantly moderated the relationship between the GMFM-66 and the selfcare domain of the PEDI-FSS. Meanwhile, many items in the self-care domain such as using the toilet, washing the face or body and wearing trousers require considerable gross motor function as well as fine motor function. In our study, GMFM-66 scores explained $49.7 \%$ of the PEDI-FSS self-care scores. This was lower than a Swedish study which reported $73.0 \%$ explanation [11]. Some of the difference in these results is likely due to a translation 
gap, such as 'puree' being translated to 'porridge' and 'unfastened shoes' being translated as a state in which shoestrings have been loosened or indicating shoes from which the Velcro has been detached. It is also important to consider that the self-care ability of children with $\mathrm{CP}$ is affected by the sociocultural attitudes involved in caring for CP children for activities such as spoon-feeding or face-washing carried out by the caregivers.

There are several limitations of the current study to be addressed. First, the number of recruited children with GMFCS level V, BFMF level V, and the ataxic and dyskinetic types of $\mathrm{CP}$ were relatively insufficient for statistical analysis. Second, we chose to focus on the relationship between gross motor function and daily functional skill, with severity, neuromotor type, distribution, and hand function as moderating factors. However, daily functional skills are affected by many other factors such as children's motivation, age, cognitive and socioeconomic variables, and environmental and cultural factors. We were limited to explaining the daily functional skill by gross motor function and fine motor function only. Further study which includes various related factors should be investigated in Korean children with $\mathrm{CP}$.

In Korean children with CP aged between 4 to 7 years, the GMFM- 66 explained not only the mobility domain of the PEDI but also some aspects of the domains of selfcare and social function. The distribution of CP and BFMF levels moderated the relationship between the GMFM-66 and the self-care domain of the PEDI-FSS. In addition, the distribution of $\mathrm{CP}$ moderated the relationship between the GMFM- 66 and the mobility domain of the PEDI-FSS. In particular, the GMFM-66 showed lower explanatory power in unilateral spastic CP than in bilateral spastic CP. This result should be considered when evaluating children with unilateral spastic CP. Although motor function as evaluated in a standardized environment showed a strong relationship with daily functional skill in Korean children with CP, these results were not completely equivalent and showed a weaker relationship compared to that indicated by foreign reports. Therefore, evaluations of daily functional skill should be included for more accurate comprehension of the realities of a child's daily life. The distribution of $\mathrm{CP}$ and hand function should be considered alongside motor function for goal-setting and enhancement of children's life skills.

\section{CONFLICT OF INTEREST}

No potential conflict of interest relevant to this article was reported.

\section{REFERENCES}

1. The Definition and Classification of Cerebral Palsy. Dev Med Child Neurol 2007;49(s109):1-44.

2. Rosenbaum P, Stewart D. The World Health Organization International Classification of Functioning, Disability, and Health: a model to guide clinical thinking, practice and research in the field of cerebral palsy. Semin Pediatr Neurol 2004;11:5-10.

3. Bax MC. Terminology and classification of cerebral palsy. Dev Med Child Neurol 1964;6:295-7.

4. Russell DJ, Rosenbaum PL, Avery LM, Lane M. Gross Motor Function Measure (GMFM-66 \& GMFM-88): user's manual. London: Mac Keith Press; 2002.

5. Haley SM, Coster WJ, Ludlow LH, Haltiwanger JT, Andrellos PJ. Pediatric Evaluation of Disability Inventory (PEDI): development, standardization and administration manual. Boston, MA: PEDI Research Group, New England Medical Center Hospital; 1992.

6. Nordmark E, Orban K, Hagglund G, Jarnlo GB. The American Paediatric Evaluation of Disability Inventory (PEDI). Applicability of PEDI in Sweden for children aged 2.0-6.9 years. Scand J Rehabil Med 1999;31:95-100.

7. Custers JW, Wassenberg-Severijnen JE, Van der Net J, Vermeer A, Hart HT, Helders PJ. Dutch adaptation and content validity of the 'Pediatric Evaluation of Disability Inventory (PEDI)'. Disabil Rehabil 2002;24:250-8.

8. Smits DW, Gorter JW, Ketelaar M, Van Schie PE, Dallmeijer AJ, Lindeman E, et al. Relationship between gross motor capacity and daily-life mobility in children with cerebral palsy. Dev Med Child Neurol 2010;52:e60-6.

9. Holsbeeke L, Ketelaar M, Schoemaker MM, Gorter JW. Capacity, capability, and performance: different constructs or three of a kind? Arch Phys Med Rehabil 2009;90:849-55.

10. Tieman BL, Palisano RJ, Gracely EJ, Rosenbaum PL. Gross motor capability and performance of mobility in children with cerebral palsy: a comparison across home, school, and outdoors/community settings. 
Phys Ther 2004;84:419-29.

11. Ostensjo S, Carlberg EB, Vollestad NK. Motor impairments in young children with cerebral palsy: relationship to gross motor function and everyday activities. Dev Med Child Neurol 2004;46:580-9.

12. Jeong BL, Yoo EY, Jung MY, Chung BI. Adaptation of the Korean-translated version of the Pediatric Evaluation of Disability Inventory and cross-cultural research: preliminary study. J Korean Soc Occup Ther 2009;17:121-32.

13. Beckung E, Hagberg G. Neuroimpairments, activity limitations, and participation restrictions in children with cerebral palsy. Dev Med Child Neurol 2002;44:309-16.

14. Arnould C, Penta M, Thonnard JL. Hand impairments and their relationship with manual ability in children with cerebral palsy. J Rehabil Med 2007;39:708-14.

15. Han T, Gray N, Vasquez MM, Zou LP, Shen K, Duncan B. Comparison of the GMFM-66 and the PEDI Functional Skills Mobility domain in a group of Chinese children with cerebral palsy. Child Care Health Dev 2011;37:398-403.

16. Fennell EB, Dikel TN. Cognitive and neuropsychological functioning in children with cerebral palsy. J Child Neurol 2001;16:58-63.

17. Ahn AR, Park HS, Cha SE, Shin WH, Cho EH, Kim EH, et al. Correlation between manual ability classification system and self-care skills in children with spastic cerebral palsy. J Korean Soc Occup Ther 2011;19:1322.

18. Kuijper MA, van der Wilden GJ, Ketelaar M, Gorter JW. Manual ability classification system for children with cerebral palsy in a school setting and its relationship to home self-care activities. Am J Occup Ther 2010;64:614-20.

19. Boyd RN, Morris ME, Graham HK. Management of upper limb dysfunction in children with cerebral palsy: a systematic review. Eur J Neurol 2001;8 Suppl 5:150-66. 
Appendix 1. Bimanual Fine Motor Function

\begin{tabular}{|c|c|c|}
\hline Level & & Function \\
\hline \multirow[t]{2}{*}{ I } & & One hand; manipulates without restrictions \\
\hline & & The other hand: manipulates without restrictions or limitations in more advanced fine motor skills \\
\hline \multirow[t]{3}{*}{ II } & (a) & One hand: manipulates without restrictions \\
\hline & & The other hand: only ability to grasp or hold \\
\hline & (b) & Both hands; limitations in more advanced fine motor skills \\
\hline \multirow[t]{4}{*}{ III } & (a) & One hand: manipulates without restrictions \\
\hline & & The other hand no functional ability \\
\hline & (b) & One hand: limitations in more advanced fine motor skills \\
\hline & & The other hand: only ability to grasp or worse \\
\hline \multirow[t]{3}{*}{ IV } & (a) & Both hands: only ability to grasp \\
\hline & (b) & One hand: only ability to grasp \\
\hline & & The other hand: only ability to hold or worse \\
\hline $\mathrm{V}$ & & Both hands: only ability to grasp or worse \\
\hline
\end{tabular}

Adapted with permission from John Wiley \& Sons Ltd. [13]. 